\title{
On the Determination of Soot and Droplet Concentration and Velocity Fields during the Addition of a Water Mist on a Liquid Pool Fire at Laboratory Scale
}

\author{
JEROME RICHARD, JEAN-PIERRE GARO, JEAN-MICHEL SOUIL \\ and JEAN-PIERRE VANTELON \\ Laboratoire de Combustion et de Détonique \\ Ecole Nationale Supérieure de Mécanique et d'Aérotechnique \\ University of Poitiers \\ BP 40109, Téléport 2, \\ 1, Avenue Clément Ader \\ 86961 Futuroscope Chasseneuil Cedex, France
}

\begin{abstract}
S
Detailed mappings are presented of extinction coefficient and flow fields at the base region of a heptane medium-scale pool fire during the addition of a water mist. These mappings were obtained by using, on the one hand, an original methodology coupling extinction measurements at two wavelengths and permitting the separation of the respective contribution of soot particles and water droplets and, on the other hand, PIV measurements and processing permitting the determination of their flow velocity patterns.

This work results in information about the interaction between mist and liquid pool fires and about the resulting structure at the base of the flame. The customarily described well defined structure of the fire flame does not exist any longer. It is observed that the size and shape vary randomly and continuously and that frequent clearances of the liquid surface, due to sudden and short vaporization periods, occur followed by extinguishment or flashback. This behavior suggests that extinguishment is achieved by total clearance of this surface rather than from the reduction of the burning rate.

The results obtained could also be used, in future work, as a data base for the prediction of the change of the radiation heat flux to the fuel surface, problem still not well known and studied. However, it is important to realize that the measurements are not instantaneous and that they provide only averaged values when the measured properties are affected by the flame disruption.
\end{abstract}

KEY WORDS : pool fire, fire suppression, water mist, radiation. 


\section{INTRODUCTON}

Water mist is now widely considered to be an interesting alternative to gaseous fire suppression agents. However, due to a lack of attention for practically two decades (1970-1990), there is still a need for better understanding of fire extinction using finely divided water. If the main mechanisms which act together -heat extraction or gas phase cooling, oxygen displacement or dilution and change of radiant heat fluxes- are now well identified [1-5], a better knowledge of each of them is essential to develop models to simulate the extinction of fire by mist and to design the appropriate extinction systems (no such models exist at this time).

Even if in all the past experiments there was no test in which radiation attenuation could be identified as the dominant mechanism of extinguishment, theoretical considerations suggest that water droplets or vapor that enter the flame will reduce the radiation heat transfer to the fuel surface, through absorption and scattering of thermal energy, and consequently reduce the evolution of flammable species. In fact, this effect is difficult to measure and more experiments are needed to make a comparison between measured and calculated radiative heat fluxes in this zone.

The thermal radiation from any combustion system is controlled by the temperature but also by the extinction coefficient distributed throughout the flame. The immediate objective of this work is to provide information about the distribution of this major property of the flame due primarily to the presence of soot particles and also to water droplets during mist addition on a burning liquid. The general flow pattern is also investigated and analyzed using PIV algorithms, permitting the characteristics of soot particles and droplets entering the flame to be deduced. This is made to infer the shape and structure of the flame during water mist addition but it should also be used to provide a data base to calculate the flame radiation in a subsequent work.

For convenience, the study is conducted with horizontal liquid fuel surface, i.e. pool fire, at laboratory scale. This has both fundamental and practical interest because it is one of the most basic forms of fuel combustion. In addition, experiments of this type are of interest because they can be conducted in the laboratory while conserving the major characteristics of an actual fire.

A large number of studies have been performed on the characteristics of buoyant diffusion flames established at the surface of small or medium-scale liquid pools [6-8]. Recall that the fire plume is customarily divided into three regions. At the flame base is the « persistent » zone which is characterized by a luminous annular flame that surrounds a central zone rich in vapor fuel and which appears, at least statistically, to have a constant shape and structure (in fact the flame fluctuates back and forth across a mean position). It is followed by the « intermittent » zone that has a turbulent character giving rise periodically to rising rolling structures. The last region is the " plume » zone that is no more reacting and where the velocity and temperature decrease progressively with height. In summary, it is possible to say that the base of the fire is characterized by a fluctuating laminar diffusion flame that is transformed into a turbulent one in the upper region. Of these zones, the persistent zone has chemical reactions and air entrainment taking place and, therefore, is the most interesting regarding flame establishment and stabilization.

Detailed measurements are then presented of the extinction coefficient and flow fields at the base region of a heptane pool fire without and with water mist addition. This is very 
important for understanding the influence of this addition on flame structure and characteristics but also on the potential impact of the fire on the fuel surface.

\section{EXPERIMENTS}

Experiments were performed with heptane pool fires of moderate scale. Heptane was chosen because it has the advantage of a fixed boiling point $\left(98^{\circ} \mathrm{C}\right)$ below that of water, avoiding then any serious splashing effect of water droplets strinking the surface which is an effect non suitable for accurate measurements. In addition, due to its low flash point temperature $\left(-4^{\circ} \mathrm{C}\right)$ and high vapor pressure, it is difficult to reduce the vapor/air mixture to below its lean flammability limit. Thus, complete extinguishment does not occur immediately, or even is not achieved, giving enough time for experimental purposes.

Heptane was contained in a circular steel pan, $10 \mathrm{~cm}$ deep, with a diameter of $23 \mathrm{~cm}$. Before each test the pan was filled to $1 \mathrm{~mm}$ below the pan lip with fresh fuel. During the test, the level was kept constant by means of a gravity liquid feeding system based on an electronically controlled on/off valve, widely used previously [9]. The rate of mass loss was $0.66 \pm 0.05 \mathrm{~g} / \mathrm{s}$ and the rate of heat release $39 \mathrm{~kW}$, providing that combustion is complete.

\section{-Water mist characteristics and application}

It is well established that the success in extinguishing liquid fires with water mist is very sensitive to the way in which the mist is applied. It requires droplets distributed with enough energy to penetrate the flame and mix turbulently and, as much as possible, uniformly in all zones of the flame. Investigations have been carried out to determine the influence of the direction of mist application. If mist is applied horizontally, the flame is deflected and the rate of air entrainment into the plume is increased. This tends to promote combustion, but there is practically no penetration of droplets into the flame and no extinction can be obtained. It is only for angles greater than about $60^{\circ}$ (opposed flow configuration) that mist becomes to be efficient.

Therefore, great attention must be paid to the location and orientation of the nozzles, to their type, to mist momentum and to mass of water added but also to droplet size distribution. The water mist system used consists of three nozzles located symmetrically with respect to the flame axis $\left(120^{\circ}\right)$ and directed toward the fuel surface center. The distance between the nozzles and the liquid surface is $129.5 \mathrm{~cm}$ and their radial position $70 \mathrm{~cm}$ (see Fig.1). The nozzles are twin fluid (water/air) pressure assisted atomizers permitting the control of the droplet size, flow density, and momentum of the mist. For purposes of comparing mist produced by the nozzles operating at varying air pressure and water flow conditions, the classical approach of reference point selection for the measurements was adopted. Although unavoidably arbitrary, the procedure is intended to provide a useful means of characterization. 


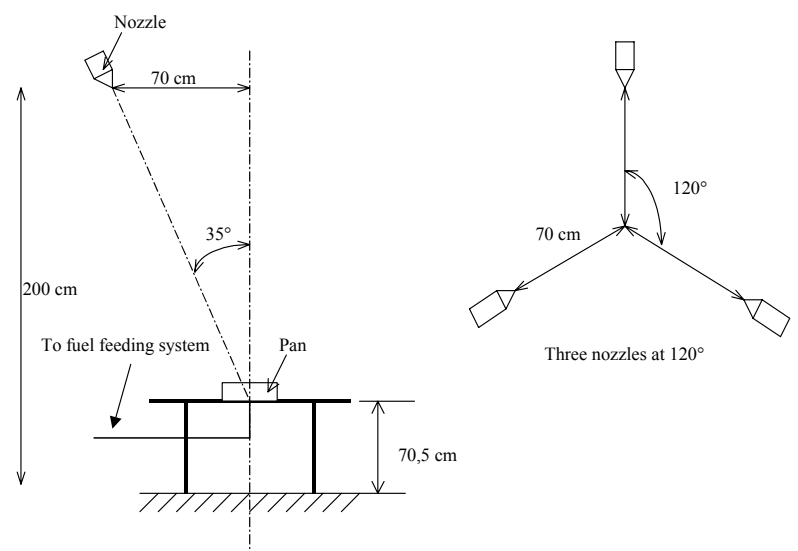

Figure 1 : Relative fire-nozzles location.

As stated above, the applied air pressure and water flow are chosen in such a way that complete extinguishment does not occur immediately or even does not occur at all. In the described experiments, each nozzle works at a normal operating air-pressure of 1 bar and provides a water mass flow rate of $3.5 \mathrm{~g} / \mathrm{s}$. For such conditions, the velocity and the mass flux of the droplets become nearly uniform $\left(2.4 \mathrm{~m} / \mathrm{s}\right.$ and $\left.0.11 \mathrm{~kg} / \mathrm{m}^{2} / \mathrm{s}\right)$ at a distance of 0.7 $\mathrm{m}$ from the tip of each nozzle. The diameter of the flow is then about $25 \mathrm{~cm}$ and the mean drop size (maximum of the unimodal distribution characterized by a phase-Doppleranemometer, PDA) is $30 \mu \mathrm{m}$..

\section{- Extinction coefficient}

Local monochromatic extinction coefficients were measured with a laser light attenuation method. A laser beam is directed to a specified location in the flame through a stainless steel tube $1 \mathrm{~cm}$ in diameter and the attenuated intensity light, passing through a second stainless steel tube of same diameter, is received by an appropriate detector which has narrow band pass filter in front of it centered at the wavelength of the emitting beam. Comparison of the light intensity through a fixed path length, between the two tubes ( 2 $\mathrm{cm}$ ), and the intensity of the emitting beam, permits the deduction of the local value of the monochromatic extinction coefficient $\mathrm{k}_{\mathrm{e}, \lambda}$ by using Beer's Law.

The whole system was mounted on a two dimensional traverse mechanism which allowed positioning in both horizontal and vertical directions to within $1 \mathrm{~mm}$. The data were time averaged. No measurements were performed for distances closer than $3 \mathrm{~cm}$ to the fuel surface because of the dimensions of the laser probe.

\section{-Velocity measurements by PIV by using a direct intercorrelation algorithm}

The laser used was a double pulse yag laser $(\lambda=532 \mathrm{~nm})$ giving $25 \mathrm{~mJ} /$ pulse in $10 \mathrm{~ns}$. The laser sheet, $2 \mathrm{~mm}$ thick, was directed along a radial vertical plane of the pan. Pictures were obtained by using a high resolution CCD video camera (1300 x 1300 pixel $\left.^{2}\right)$ fitted with a lens having a $50 \mathrm{~mm}$ focal length (f/1.2) providing a field of view of $25 \times 30 \mathrm{~cm}$. The time between two successive pictures was $2 \mathrm{~ms}$.

With water mist addition spots due to both water droplet and soot particle scattering are evidenced (even if in most instances, due to the small size of soot particles, scattering has 
little effect on the emittance of these particles in the wavelength range at hydrocarbon combustion temperatures). A typical example of a picture is given in Fig 2 (a). To obtain information about the dynamics of the droplets alone, an original algorithm permitting the spots to be isolated was developed.

Consider a matix $I_{0}(m, n)$ (dimension $M \times N$ ) representative of a picture of the flame interacting with the water mist. Pre-processing of the image consists of successive application of three operators $\mathrm{Op}_{1}, \mathrm{Op}_{2}$ and $\mathrm{Op}_{3}$ :

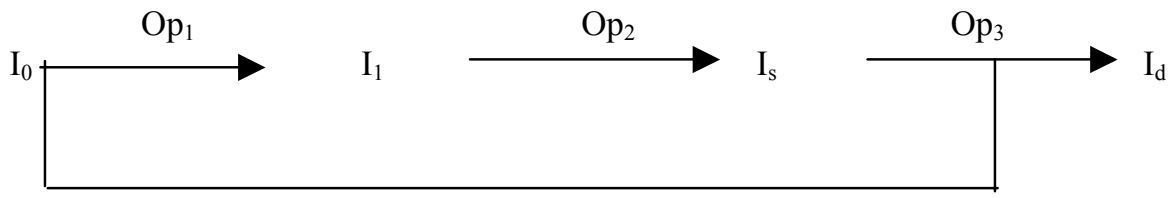

The operator $\mathrm{Op}_{1}$ is defined by :

$$
\begin{array}{r}
\mathrm{Op}_{1}\left(\mathrm{I}_{0}\right)=\mathrm{I}_{1} \text { with } \mathrm{I}_{1}(\mathrm{~m}, \mathrm{n})= \\
\mathrm{min}\left[\mathrm{I}_{0}(\mathrm{i}, \mathrm{j})\right] \\
\mathrm{m}-1 \leq \mathrm{i} \leq \mathrm{m}+1 \\
\mathrm{n}-1 \leq \mathrm{j} \leq \mathrm{n}+1
\end{array}
$$

The objective of this first step is to eliminate the scattering spots due to the water droplets from the initial picture. Indeed, the contrast being important and the area of the spots being less than 9 pixels ${ }^{2}$, these spots are substituted by black pixels. But this step has also for consequence to delete the spots due to soot. In order to reinstitute them in their initial form, the operator $\mathrm{Op}_{2}$ is applied :

$$
\begin{aligned}
\mathrm{Op}_{2}\left(\mathrm{I}_{1}\right)=\mathrm{I}_{\mathrm{s}} \text { with } \mathrm{I}_{\mathrm{s}}(\mathrm{m}, \mathrm{n})= & \operatorname{Max}\left[\mathrm{I}_{1}(\mathrm{i}, \mathrm{j})\right] \\
& \mathrm{m}-1 \leq \mathrm{i} \leq \mathrm{m}+1 \\
& \mathrm{n}-1 \leq \mathrm{j} \leq \mathrm{n}+1
\end{aligned}
$$

The deduced picture (Fig.2 (b)) is similar to the initial but without the scattering spots due to the droplets. By substracting this second picture from the initial one, a final picture is obtained (Fig.2 (c)) showing only the scattering spots due to the water droplets. This last operation is expressed by :

$$
\mathrm{Op}_{3}\left(\mathrm{I}_{0}, \mathrm{I}_{\mathrm{s}}\right)=\mathrm{I}_{\mathrm{d}} \text { with } \mathrm{I}_{\mathrm{d}}(\mathrm{m}, \mathrm{n})=\mathrm{I}_{0}(\mathrm{~m}, \mathrm{n})-\mathrm{I}_{\mathrm{s}}(\mathrm{m}, \mathrm{n})
$$

and was applied to all the pictures involving water mist addition.

The PIV calculation used was based on a direct intercorrelation algorithm, previously developed in this Laboratory by Susset et al. [10]. This algorithm was applied to pictures $I_{s}$ and $I_{d}$. 


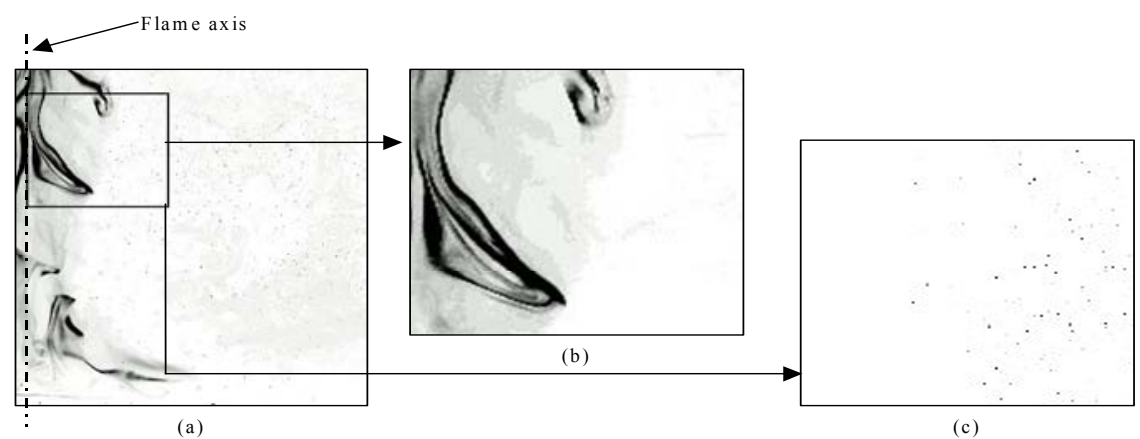

Figure 2 : Example of image processing. (a) Initial image, (b) $I_{s}$ image and (c) $I_{d}$ image (extended).

\section{RESULTS AND DISCUSSION}

The characteristics of the flame without water mist addition constitutes a reference for the present work.

When water mist is added with the chosen nozzle system configuration, the flame structure changes completely. The interaction between mist and flame can be divided into three phases. During the first phase, the behavior of the flame does not really differ very much from its behavior before mist application. The flame only seems to be pushed against the fuel surface and its volume is significantly reduced, especially at the top. During the second phase, the flame suddenly seems "explode" with random lateral projection of more or less large pockets of warm unburnt fuel and products. This is the result of penetration of droplets, distributed with enough energy to cause them to mix turbulently in the flame, and of their rapid evaporation and expansion which causes gas displacement in their vicinity. During this phase of lateral expansion, clearance from part or even the whole of the liquid surface appears. The flame looks like an annular flame anchored around the inside edge of the pan. It is noteworthy that it is during this phase that the extinction can be obtained. This requires that the mist be applied with enough momentum so as to overcome the anchoring effect and to avoid flash back. If extinction is not achieved, in a third phase the flame is restructured, recovering its first phase behavior. Afterwards, a new cycle can take place. An analysis of simultaneous video recording shows that, on the whole, the time between two expansion phases is approximately $4 \mathrm{~s}$ and that the expansion time is within $0.5 \mathrm{~s}$.

It is noteworthy that this periodical behavior is not observed over a broad spectrum of conditions. As stated above, the configuration must be an opposed flow configuration. In addition, if the mass flux and velocity of the droplets are too low, there is not enough penetration and mixing. If they are too elevated, the flame is flattened against the surface and can be suppressed by the mechanism of blow out, which is not the expected effect. In fact, the phenomenon takes place for a limited range of conditions, its magnitude and frequency being mainly linked to the applied air-pressure. The water mass flow rate and the drop size play also a role but to a lower extend.

Flame before water mist addition (reference flame) 
The measurements were performed along a radial vertical plane at the base of the heptane pool fire. Because the flame characteristics in this zone are statistically axisymmetric the measurements are considered to be representative of those in any other radial vertical plane.

\section{-Extinction measurements}

Measurements of extinction coefficients were performed in the visible range of the spectrum with a helium/neon laser emitting at $633 \mathrm{~nm}$, wavelength at which the extinction is due primarily to the presence of soot. Due to their small size $(60-80 \mathrm{~nm})$, the soot particles are mainly absorbing particles (scattering is negligible) and the actual coefficients are absorption coefficients, $\mathrm{k}_{\mathrm{a}, \lambda}$, rather than extinction coefficients. Thence, the contour of the lines can also be viewed as lines of equal soot concentration (Fig.3a). The measurements show a region of elevated soot concentration at the axis. In the region near the axis and under the maximum temperature line, the existence of stagnant and recirculating gas would result in the accumulation of fuel vapor, pyrolysis products and soot. Soot concentration peaks also near the pool surface centerline as generally observed. Above the temperature maximum, the presence of elevated soot concentration at the centerline indicates the presence of unburnt fuel and corresponds to the continuation, even to a lower extent, of the combustion reaction in the intermittent zone of the flame.

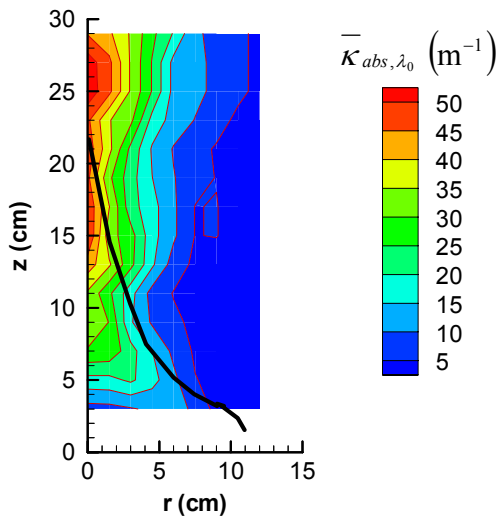

(a)

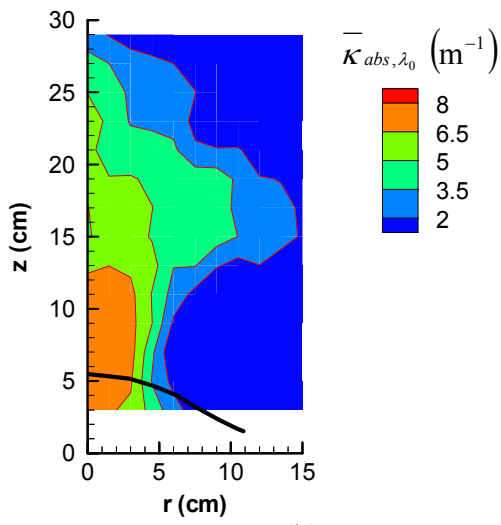

(b)

Figure 3 : a- Field of monochromatic absorption coefficient $(\lambda=633 \mathrm{~nm})$ due to soot at the base of the heptane pool fire before water mist addition (the line of mean temperature maximum is also indicated for reference).

b- Field of monochromatic absorption coefficient $(\lambda=633 \mathrm{~nm})$ due to soot at the base of the heptane pool fire during water mist addition (the line of mean temperature maximum is also indicated for reference).

\section{- PIV measurements}

The overall flow velocity field is shown in Fig.4a. The soot particles are small enough to make the assumption that the calculated velocities are representative of the gaseous flow velocity. Therefore, from the observed trend of this field, the major characteristics of the 
structure of the flame are evidenced: chemical reaction and air entrainment near the fuel surface and upward movement of the gases due to effects of buoyancy as a result of temperature gradients.

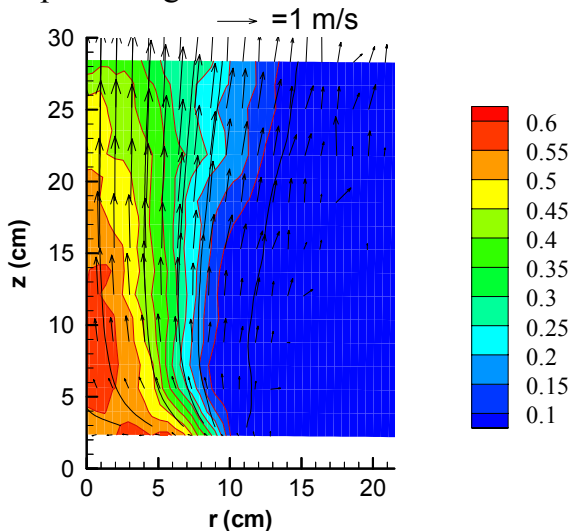

(a)

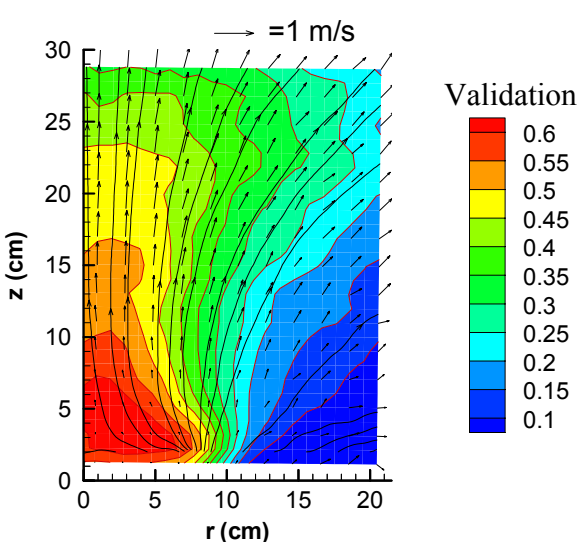

(b)

Figure 4 : a- Soot velocity field and validation criterion at the base of the heptane pool fire before water mist addition.

b- Soot velocity field and validation criterion at the base of the heptane pool fire during water mist addition.

However, there is no experimental evidence of the existence of the wellknown stagnation recirculation zone in the central region, close to the fuel surface. The cause is that the measurements of the flow field are limited in zones too high above the surface for technical reasons. The variable shown by color level in this figure is a validation criterion. It is defined as the local ratio of the number of vectors validated by the PIV algorithm to calculate the soot mean velocity on the total number of flow field computed. This variable may be viewed as a soot presence probability rate. It is seen that both absorption coefficient (i.e. soot concentration) in Fig. 3a, and soot presence probability fields show very similar patterns.

\section{Flame with water mist addition}

Despite the successive cycles of disruption and reconstruction, the flame with water mist addition can also be considered to be statistically axisymmetric and the present measurements were also limited to a radial vertical plane.

\section{-Extinction measurements}

With water mist, in addition to soot particles, water droplets contribute to some extent to attenuation of the radiant heat through absorption but also through scattering. For soot particles, we have seen that scattering can be ignored due to their small size, but for water droplets, whose dimension is much more larger, scattering becomes rapidly comparable to absorption or even predominant. Droplets may scatter in nearly isotropic manner if small enough, or forward in such a way that the bulk of the scattered radiation is 
contained in a narrow solid angle around the incident direction when the droplets are relatively large.

Let us consider a detector of surface $\mathrm{S}$ centered on an incident beam axis at a given distance from a droplet. The radius of the detector and its location determine the collected scattered power in the forward direction. In terms of absorption and scattered efficiencies, $\mathrm{Q}_{\mathrm{a} \lambda}$ and $\mathrm{Q}_{\mathrm{s} \lambda}$, an apparent monochromatic extinction coefficient $\mathrm{k}_{\mathrm{e} \lambda}^{\prime}$ can be written :

$$
\mathrm{k}_{\mathrm{e} \lambda}^{\prime}=\mathrm{sN}\left(\mathrm{Q}_{\mathrm{a} \lambda}+\mathrm{Q}_{\mathrm{s} \lambda}\left(1-\mathrm{R}_{\lambda}\right)\right) \quad \text { or } \quad \mathrm{k}_{\mathrm{e} \lambda}^{\prime}=\mathrm{k}_{\mathrm{a} \lambda}+\mathrm{k}_{\mathrm{s} \lambda}\left(1-\mathrm{R}_{\lambda}\right)
$$

where $\mathrm{s}$ is the droplet cross section, $\mathrm{N}$ is the droplet concentration and $\mathrm{R}_{\lambda}$ is the normalized collected scattered power for the detector used, dependent on its location with respect to the path length, the droplet diameter and the wavelength.

For experiments performed in a medium containing both soot particles and water droplets, the apparent extinction coefficient is then written as follows :

$$
\mathrm{k}_{\mathrm{e}}^{\prime}=\mathrm{k}_{\mathrm{as}}+\mathrm{k}_{\mathrm{ad}}+\mathrm{k}_{\mathrm{sd}}(1-\mathrm{R})=\mathrm{k}_{\mathrm{as}}+\mathrm{k}_{\mathrm{sd}}\left[(1-\mathrm{R})+\mathrm{Q}_{\mathrm{ad}} / \mathrm{Q}_{\mathrm{sd}}\right]
$$

where the second subscripts $\mathrm{s}$ and $\mathrm{d}$ mean respectively pertaining to soot and water droplet (subscript $\lambda$ is omitted to simplify the writing) and where the absorption and scattering efficiencies, $\mathrm{Q}_{\mathrm{ad}}$ and $\mathrm{Q}_{\mathrm{sd}}$, can be calculated from the Mie theory since they are independent of particle concentration and medium depth, provided the assumption of single scattering holds.

Calculations have been performed considering, as stated earlier, a monodisperse water droplet suspension with a diameter of $30 \mu \mathrm{m}$ and, likewise, a monodisperse soot particle suspension with a diameter of $70 \mathrm{~nm}$. The value of refractive index used are, for soot particles those proposed by Chang and Charalampopoulos [11], and for water droplets those proposed by Hale and Querry [12].

The concentrations of soot and water droplets being unknown, it is clear evidence that this relation is not sufficient to determine the respective contribution of soot and droplets to the detector signal. Thus, it was necessary to perform measurements at another wavelength. In order to minimize the forward scattering of water droplets, the wavelength was chosen in the infrared range: a helium/neon laser emitting at $3390 \mathrm{~nm}$ (notice that there is no contribution of gaseous combustion products at this wavelength).

In addition, a relation between the measurements at the two wavelengths is needed. For example, the ratio between the soot absorption coefficients at the two wavelengths, which is equal to the ratio of the corresponding absorption efficiencies, can be used.

The field of monochromatic soot absorption coefficient $(\lambda=633 \mathrm{~nm})$ with water mist addition is presented in Fig.3b. It is seen that the values are, on the whole, much more lower than without mist addition. The region of more elevated soot concentration is always near the pool axis, but it is pushed against the fuel surface. Above this region, the general pattern is expended. This is essentially due to the periodic strong radial convective component induced by the expansion effect of mist evaporation. The reduction of soot concentration suggests that mist addition should have an important role in the combustion process: mixing and chemical effects. Water addition disturbs the flow, the temperature and the fuel concentration field; this greatly affects both chemical and physical phenomena. The expansion effect due to droplets vaporization tends to increase 
mixing and turbulence and the combustion efficiency is increased. But, especially, the strong entrainment and penetration of $\mathrm{O}_{2}$ over most of the regions and the probable presence of $\mathrm{OH}$ radicals, leads also to a decrease of the yield of soot. Figure 5 shows the field of monochromatic droplet scattering coefficient $(\lambda=633 \mathrm{~nm})$. It reveals an expanded zone, like a "dome", of higher concentrations, especially in the upper region. This result is logical since the injection of finely divided water mist into a flame results in rapid droplets evaporation. Droplets can be present in noticeable quantity only at the periphery. Next, they evolve as they move into the flame; water vapor created is pushed in the inner region of the flame, onto the fuel surface. When this surface is approached, no more droplets are detected, especially closer to the pool centerline.

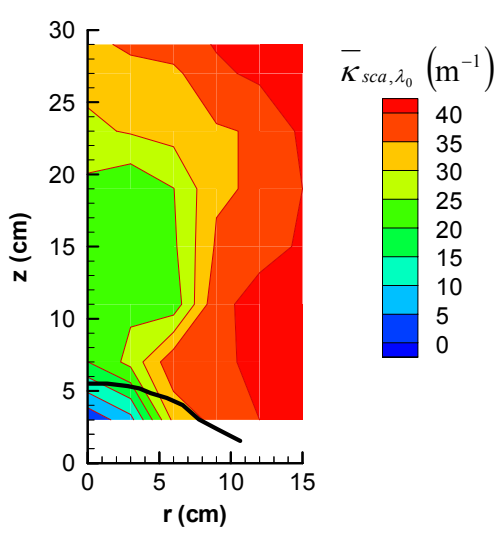

Figure 5 : Field of monochromatic scattering coefficient $(\lambda=633 \mathrm{~nm})$ due to water droplets at the base of the heptane pool fire during water mist addition.

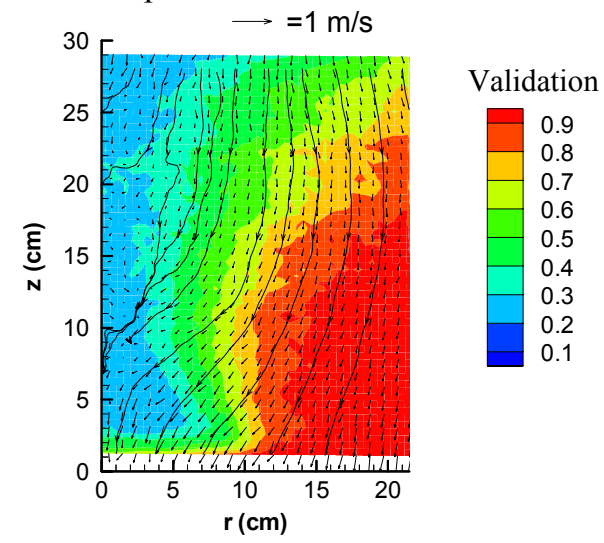

Figure 6 : Droplet velocity field and validation criterion at the base of the heptane pool fire during water mist addition.

\section{-PIV measurements}

\section{-Soot correlation}

It can be seen in Fig. $4 \mathrm{~b}$ that the general soot flow pattern is no more representative of a fire plume but rather of a counter-diffusion system with its inward direction near the surface that turns upward in the upper regions. As stated above, the periodic expansion of the flame gives rise to an increase of the radial component of the velocity and, therefore, to an inflection of the corresponding lines that turn in the outward direction. Soot concentration shown as a probability contour by the validation criterion, defined above, can be compared with the absorption coefficient field in Fig.3b. These fields are in essential agreement: a zone of more elevated soot concentration at the axis, near the fuel surface, and above an expanded zone of lower concentrations that coincides with the flow lines turning outward.

\section{-Droplet correlation}

The overall flow velocity field of water droplets is shown in Fig.6. It is seen that the direction of the velocity lines is deflected outward due to the conjunction of two effects:

- the effect of the upward motion of the gases generated by buoyancy that deflects the water droplets from their initial direction, 
- the "puffs", described above, that add an important positive radial component which compensates partially the negative radial component of the initial velocity.

There is a zone where the droplet velocity is very low. This zone is located near the axis at about $15 \mathrm{~cm}$ above the fuel surface and corresponds to the area where the initial momentum of the droplets is annihilated by the rising of the hot gases and by the radial component added by the "puffs".

In Fig.6, the validation criterion is also reported by color level. Three distinct zones are evidenced. The first one is on the top of the investigated region and surrounds the flame. In this zone, the droplet concentration is maximum. The second one corresponds to a central zone near the axis where the droplet concentration is minimum. Between, these two zones, the third one is a transition zone where the droplet concentration decreases with the distance to the axis. The general concentration pattern results from the effects of two phenomena :

- the mean fluid movement which is determined by the coupling of the momentum of the mist, the upward motion of the flame gases and the "puffs", as explained above,

- the effect of the temperature gradient between the surrounding and the flame that generates a force that tends to push the droplets out of the flame zone.

Here again, the general flow pattern and the deduced probability contours for droplets to be present at different locations, are in essential agreement with the contours of constant monochromatic scattering coefficient which can also be viewed as lines of equal droplet concentration.

\section{CONCLUDING REMARKS}

The detailed mapping obtained in this work of the velocity and soot and water droplet concentration has resulted in new information about the flame structure at the base of a hydrocarbon (heptane) pool fire, at laboratory scale, during water mist addition.

An important result is the realization that unlike a steady flame for which the structure is determined by the coupling between thermal, chemical and aerodynamic processes, the strong radial convective component, induced by the expansion effect of sudden mist vaporization decorrelates completely the phenomena and, then, modifies the structure of the whole flame. The shape of the flame varies continuously and its size is reduced. There are periodic clearances from part or the whole surface, extinguishment being sometimes obtained after one of these sudden clearances of the flame. This behavior suggests that extinguishment is rather achieved by this rapid and total clearance, resulting from water vaporization and expansion effect, than from the reduction of the rate of evolution of combustible vapor from the liquid surface. Moreover, fuel vapor is probably pushed against this surface with subsequent increase in concentration. The remark is especially important for hydrocarbons with low flash points such as heptane : flash back may occur easily and any hot object near the fuel surface, such as the pan edge, can cause re-ignition.

Although the change of radiant heat transfer is not recognized as a dominant effect in hydrocarbon fire extinguishment, a better understanding of this effect is essential for any attempt of modelling development. Soot is known to be the most important radiating product formed when burning hydrocarbons and the addition of water droplets into the flame complicates the radiation transfer. Account for the effect of soot or water droplets on flame radiation implies to know their concentration distribution into the flame. From 
this standpoint, the present results constitute also a data base for subsequent radiative attenuation computation.

\section{Acknowledgement}

The authors express their thanks to Total Fina Elf Company for its interest and help in this study.

\section{REFERENCES}

[1] Braidech, M.M., Neale, J.A., Matson, A.F., and Dufour, R.E., The mechanism of extinguishment of fire by finely divided water, Underwriters Laboratories Inc. For the National Board of Fire Underwriters, N.Y., 1955, p.73.

[2] Rasbash, D.J., Rogowski, Z.W.,and Stark, G.W.V., "Mechanisms of extinction of liquid fuel fires with water sprays", Comb. and Flame, 4: 223-234 (1960).

[3] Rasbash, D.J., " The extinction of fires by water sprays”, Fire Res. Abstr.Rev.,4: 223-234 (1962).

[4] Rasbash, D.J., "The extinction of fires with plain water : a review", Fire Safety ScienceProceedings of the First International Symposium, International Association for Fire Safety Science, Interscience Communications, London, UK, 1986, pp.1145-1163.

[5] Mawhinney, J.R., Dlugogorski, B.Z., and Kim, A.K., "A closer look at the fire extinguishing properties of water mist", Fire Safety Science-Proceedings of the Fourth International Symposium, International Association for Fire Safety Science, Interscience Communications, London, UK, 1994, pp.47-60.

[6] McCaffrey, B.J., "Purely buoyant diffusion flames. Some experimental results". Center for Fire Research National Bureau of Standards, Report NBSIR 1910, (1979).

[7] Bouhafid, A., Vantelon, J.P., Joulain, P., and Fernandez Pello, A.C., "On the flame structure at the base of a pool fire", Proceeding of the Twenty Second Symposium (International) on Combustion, The Combustion Institute, Pittsburgh,1988, pp.1291-1298.

[8] Venkatesh, S., Ito, A., Saito, K., and Wichman, I.S., "Flame base structure of a smallscale pool fires", Proceeding of the Twenty Sixth Symposium (International) on Combustion, The Combustion Institute, Pittsburgh, 1996, pp.1437-1443.

[9] Souil, J.M., Vantelon, J.P., Joulain, P., and Grosshandler, W.L., "Experimental and theoretical study of thermal radiation from freely burning kerosene pool fire", Prog in Astro and Aeronautics, Vol.105, Part.1 : "Flames and Configuration", 1986, pp.388-401.

[10] Susset, A., Most, J.M., Honoré, D., and Perrin, M., "Développement d'un traitement itératif par corrélation directe pour l'application de la PIV aux écoulements à forts gradients de vitesse", Septième Congrès de l'AFVL, Marseille, France, 19-22 Septembre 2000.

[11] Chang, H., and Charalampopoulos, T.T., "Determination of the wavelength dependence of refractive indices of flame soot", Proc. R. Soc. Lond, A, 430: 576-587 (1990).

[12] Hale, G.M., and Querry, R.M., "Optical constants of water in the $200 \mathrm{~nm}$ to $200 \mu \mathrm{m}$ wave-length region”, Applied Optics, Vol 12, n³: 555-563 (1973). 